\title{
Inulin oligofructose attenuates metabolic syndrome in high-carbohydrate, high-fat diet-fed rats
}

\author{
Senthil A. Kumar ${ }^{1}$, Leigh C. Ward ${ }^{2}$ and Lindsay Brown ${ }^{1,3 *}$ \\ ${ }^{1}$ Functional Foods Research Group, University of Southern Queensland, Toowoomba, Qld 4350, Australia \\ ${ }^{2}$ School of Chemistry and Molecular Biosciences, The University of Queensland, Brisbane, Qld 4072, Australia \\ ${ }^{3}$ School of Health and Wellbeing, University of Southern Queensland, Toowoomba, Qld 4350, Australia \\ (Submitted 1 May 2016 - Final revision received 4 August 2016 - Accepted 16 September 2016)
}

\begin{abstract}
Prebiotics alter bacterial content in the colon, and therefore could be useful for obesity management. We investigated the changes following addition of inulin oligofructose (IO) in the food of rats fed either a corn starch (C) diet or a high-carbohydrate, high-fat (H) diet as a model of diet-induced metabolic syndrome. IO did not affect food intake, but reduced body weight gain by $5 \cdot 3$ and $12 \cdot 3 \%$ in corn starch + inulin oligofructose (CIO) and high-carbohydrate, high-fat with inulin oligofructose (HIO) rats, respectively. IO reduced plasma concentrations of free fatty acids by $26 \cdot 2 \%$ and TAG by $75.8 \%$ in HIO rats. IO increased faecal output by $93.2 \%$, faecal lipid excretion by $37.9 \%$ and weight of caecum by $23.4 \%$ and colon by $41.5 \%$ in HIO rats. IO improved ileal morphology by reducing inflammation and improving the density of crypt cells in HIO rats. IO attenuated H diet-induced increases in abdominal fat pads (C 275 (SEM 19), CIO 264 (SEM 40), H 688 (SEM 55 ), HIO 419

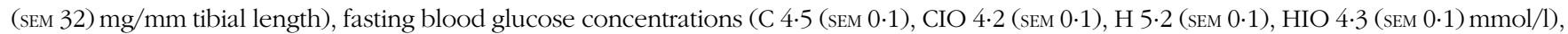
systolic blood pressure (C 124 (SEM 2), CIO 118 (SEM 2), H 152 (SEM 2), HIO 123 (SEM 3) mmHg), left ventricular diastolic stiffness

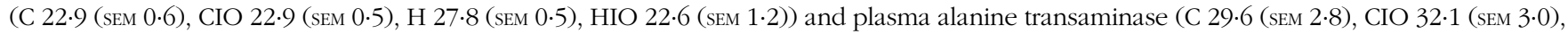
H 43.9 (SEM 2.6), HIO 33.6 (sEm 2.0) U/1). IO attenuated H-induced increases in inflammatory cell infiltration in the heart and liver, lipid droplets in the liver and plasma lipids as well as impaired glucose and insulin tolerance. These results suggest that increasing soluble fibre intake with IO improves signs of the metabolic syndrome by decreasing gastrointestinal carbohydrate and lipid uptake.
\end{abstract}

\section{Key words: Inulin: Oligofructose: Prebiotics: Metabolic syndrome: Rats}

Functional foods are differentiated from other foods by their ability to improve health and well-being or reduce the risk of disease, in addition to providing adequate nutrition ${ }^{(1,2)}$. These foods increase the fibre content of the diet and include cereals, fruits, vegetables, dried beans and lentils. These fibrecontaining foods contain fructan-type oligosaccharides such as inulin, an important dietary component since pre-historic times $^{(3)}$. Food fibre may act as a prebiotic by being resistant to gastric acidity and enzymes such as $\alpha$-glucosidase, maltase, isomaltase and sucrase, and then undergoing fermentation by bacteria in the colon to SCFA that promote the growth of Bifidobacterium and Lactobacillus species ${ }^{(4)}$. These changes in the gut bacteria are associated with prevention or postponement of CVD with hypercholesterolaemia, osteoporosis, diabetes, gastrointestinal infections and gut inflammation ${ }^{(4)}$. The WHO-recommended consumption of dietary fibre of $25 \mathrm{~g} / \mathrm{d}$ is rarely achieved in many countries, such as those with Western-style diets including the USA with an estimated average fibre intake of $15 \mathrm{~g} / \mathrm{d}^{(5)}$. Dietary consumption of inulin has been reported as $1-4 \mathrm{~g} / \mathrm{d}$ in the USA and $3-11 \mathrm{~g} / \mathrm{d}$ in Europe, below the tolerated dose of $10-20 \mathrm{~g} / \mathrm{d}^{(6)}$. Commercially, inulin is mostly extracted from chicory root, although it is present in many foods such as wheat, onions, bananas, Jerusalem artichoke and leeks; inulin has widespread uses in the pharmaceutical industry ${ }^{(7)}$. Inulin is a mixture of linear fructose polymers or fructans of two to sixty units each linked by unique $\beta^{(2-1)}$ bonds with a glucose unit linked by an $\alpha^{(1-2)}$ bond at the end of each chain ${ }^{(8,9)}$. These $\beta^{(2-1)}$ bonds cannot be hydrolysed by salivary or pancreatic enzymes as with typical carbohydrates, and therefore inulin has a reduced energy value as well as dietary prebiotic effects ${ }^{(8,9)}$. Oligofructose, a term describing fructo-oligosaccharides, refers to a hydrolysed form of inulin with two-ten monosaccharide units, possibly with a glucose terminal unit ${ }^{(8)}$. Functional foods have been studied as possible lifestyle changes to control obesity and reduce associated health risks $^{(9)}$. Obesity and dyslipidaemia are important facets of the

Abbreviations: C, corn starch; CIO, corn starch $+5 \%$ inulin oligofructose; H, high-carbohydrate, high-fat diet; HIO, high-carbohydrate, high-fat+ inulin oligofructose diet; IO, inulin oligofructose; LPS, lipopolysaccharide; LV, left ventricular; LVIDs, left ventricular internal diameter in systole; SBP, systolic blood pressure.

*Corresponding author: Professor L. Brown, email Lindsay.Brown@usq.edu.au 
metabolic syndrome. Intervention with inulin oligofructose (IO) decreased energy intake and fat mass in rats fed a high-fat diet ${ }^{(10)}$, promoted satiety ${ }^{(11,12)}$ and improved lipid metabolism, although results in humans have been less consistent ${ }^{(13)}$.

Rats fed a high-carbohydrate, high-fat diet mimic the cardiovascular, liver and metabolic changes of the metabolic syndrome in humans ${ }^{(14)}$. In this study, we have determined whether addition of IO as a dietary intervention reversed the responses to this obesogenic diet in rats. Gastrointestinal, metabolic, liver and cardiovascular parameters were measured for this comparison. Orafti (R) Synergy1 is an oligofructose-enriched inulin mixture comprised of 92 (SEM 2) \% IO as a blended mixture of equal parts of inulin and oligofructose ${ }^{(15,16)}$. Our hypothesis was that addition of IO will reverse obesity-related changes to the gastrointestinal tract leading to reversal of changes in abdominal fat pads, systolic blood pressure (SBP), heart and liver structure and function, and reduced infiltration of inflammatory cells in our rat model of the metabolic syndrome.

\section{Methods}

\section{Rats and diets}

Male Wistar rats ( $n$ 48, 8-9 weeks old; initial body weight 336 (SEM 2) g) supplied by the Animal Resource Centre in Perth, Australia, were individually housed in a temperature-controlled $\left(20 \pm 2^{\circ} \mathrm{C}\right)$, 12 -h light $-12 \mathrm{~h}$ dark cycle environment with free access to water and rat diet at the University of Southern Queensland Animal House. All the experiments were approved by the Animal Ethics Committees of The University of Queensland and the University of Southern Queensland under the guidelines of the National Health and Medical Research Council of Australia. Rats were randomly divided into four groups (twelve each) and fed corn starch (C), corn starch $+5 \%$ inulin oligofructose (CIO), a high-carbohydrate, high-fat diet $(\mathrm{H})$ and a high-carbohydrate, high-fat + inulin oligofructose diet (HIO).

Body weight and food and water intakes were measured daily, and feed efficiency (\%) was calculated ${ }^{(14,17)}$. The preparation and macronutrient composition of basal diets, including the dietary fatty acid profiles, have been detailed previously ${ }^{(14,18)}$. The IO-containing diets were prepared by adding $5 \%$ of IO replacing an equivalent amount of water in the diet. The IO diets were administered for 8 weeks, starting 8 weeks after initiation of the corn starch or high-carbohydrate, high-fat diet. Drinking water for $\mathrm{H}$ and $\mathrm{HIO}$ rats was augmented with $25 \%$ fructose for the duration of the study.

\section{Cardiovascular measurements}

SBP was measured under light sedation with Zoletil (tiletamine $15 \mathrm{mg} / \mathrm{kg}$, zolazepam $15 \mathrm{mg} / \mathrm{kg}$ by intraperitoneal injection; Virbac), using a MLT1010 Piezo-Electric Pulse Transducer and an inflatable tail-cuff connected to a MLT844 Physiological Pressure Transducer using PowerLab data acquisition unit (AD Instruments Australia and Pacific Islands) ${ }^{(14,17)}$

Echocardiographic examination (Philips/Hewlett Packard Sonos 5500, $12 \mathrm{MHz}$ transducer; Philips Healthcare) was performed at 16 weeks $^{(14,17)}$. The ventricular contractility indices were calculated, including ratio of SBP:left ventricular internal diameter in systole (LVIDs), ratio of SBP:systolic volume and ratio of end-systolic stress (ESS):LVIDs ${ }^{(14,19)}$. Left ventricular (LV) function of rats was assessed using the Langendorff heart preparation $^{(14,17)}$. Terminal anaesthesia was induced via intraperitoneal injection of pentobarbitone sodium (Lethabarb ${ }^{\circledR}, 100 \mathrm{mg}$ / kg). Heparin (200 IU; Sigma-Aldrich Australia) was administered through the right femoral vein before blood (approximately $5 \mathrm{ml}$ ) was taken from the abdominal aorta. Isovolumetric ventricular function was measured by inserting a latex balloon catheter into the left ventricle of the isolated heart connected to a Capto SP844 MLT844 physiological pressure transducer and Chart software on a MacLab system (AD Instruments Australia and Pacific Islands) ${ }^{(14-16)}$

Aortic contraction was determined using thoracic aortic rings (approximately $4 \mathrm{~mm}$ in length) suspended in an organ bath chamber with a resting tension of approximately $10 \mathrm{mN}$. Cumulative concentration-response (contraction) curves were measured for noradrenaline (Sigma-Aldrich Australia); concentration-response (relaxation) curves were measured for acetylcholine (Sigma-Aldrich Australia) or sodium nitroprusside (Sigma-Aldrich Australia) in the presence of a submaximal $(70 \%)$ contraction to noradrenaline ${ }^{(14,17)}$.

\section{Oral glucose and insulin tolerance tests}

For oral glucose tolerance test, basal blood glucose concentrations were measured in blood collected from the tail veins of overnight food-deprived rats using Medisense Precision Q.I.D glucose meter (Abbott Laboratories). Fructose-supplemented drinking water in the $\mathrm{H}$ and $\mathrm{HIO}$ groups was replaced with normal water for the overnight food-deprivation period ${ }^{(14,20)}$. Rats were administered $2 \mathrm{~g} / \mathrm{kg}$ body weight of glucose as a $40 \%$ aqueous solution via oral gavage. Tail vein blood samples were collected at 30, 60, 90 and 120 min following glucose administration. Insulin tolerance testing was performed after $5 \mathrm{~h}$ of food deprivation in rats administered an intraperitoneal injection of $0.33 \mathrm{IU}$ insulin $/ \mathrm{kg}$ body weight. Tail vein blood samples were collected at 15, 30, 45, 60, 90 and $120 \mathrm{~min}$ for blood glucose measurements after intraperitoneal insulin administration ${ }^{(14,20)}$.

\section{Body composition measurements}

Dual energy X-ray absorptiometric (DXA) measurements were performed on rats after 16 weeks of feeding, $2 \mathrm{~d}$ before rats were euthanised for pathophysiological assessments, using a Norland XR36 DXA instrument (Norland Corp.). DXA scans were analysed using the manufacturer's recommended software for use in laboratory animals (Small Subject Analysis Software, version 2.5.3/1.3.1; Norland Corp.) ${ }^{(14)}$. The precision error of lean mass for replicate measurements, with repositioning, was $3 \cdot 2 \%$. The visceral adiposity index (\%) was calculated ${ }^{(14,17)}$.

\section{Organ measurements}

The right and left ventricles were separated following perfusion experiments and weighed. Liver and retroperitoneal, epididymal and omental fat pads were dissected following heart 
removal and blotted dry for weighing. Organ weights were normalised relative to the tibial length at the time of their removal (in $\mathrm{mg} / \mathrm{mm})^{(14,17)}$. The stomach, small intestine, caecum and colon weight with contents were weighed and measured. Weights were normalised relative to the tibial length at the time of their removal (in $\mathrm{mg} / \mathrm{mm}$ ). The lengths of the small intestine and colon (proximal and distal colon) were measured using a standard ruler ${ }^{(21,22)}$.

\section{Lipid excretion and faecal output analysis}

After 16 weeks, C, CIO, $\mathrm{H}$ and $\mathrm{HIO}$ rats (six each) were individually housed in metabolic cages for $12 \mathrm{~h}$, with the same food and water. Faeces from individual rats were collected, weighed and stored in an airtight, sealed container at $-20^{\circ} \mathrm{C}$ for later measurements. The faecal samples were powdered using a mortar and pestle. Dietary lipids were extracted from $1 \mathrm{~g}$ of powdered faecal material by manual solvent extraction using a 2:1 chloroform-methanol mixture with $0 \cdot 1 \%$ vitamin $\mathrm{E}$ as an antioxidant. The solvent faecal matter was mixed on a rotating device for $40 \mathrm{~min}$ with $20-\mathrm{ml}$ chloroform-methanol solvent and then centrifuged at $2500 \mathrm{rpm}$ for $5 \mathrm{~min}$. The extraction procedure was repeated twice with subsequent washing with doubledistilled water to remove all polar material. Extracts were pooled, and the chloroform-methanol solution was evaporated under a stream of $\mathrm{N}_{2}$ on a hot plate at $60^{\circ} \mathrm{C}$ until the beakers reached constant weight, allowing calculation of gravimetric extractable lipid content ${ }^{(23)}$. The percentage lipid excretion was calculated using the following formula: ((amount of lipid excreted $(\mathrm{g}) /$ total amount of lipid consumed $(\mathrm{g})) \times 100)$ ). The amount of extractable lipids present in either $\mathrm{C}$ or $\mathrm{H}$ diets $\left(6 \cdot 2\right.$ and $187 \mathrm{~g} / \mathrm{kg}$ diet, respectively) was determined ${ }^{(24)}$.

\section{Histology}

Only two rats per group were allocated for histological analysis; two slides were prepared for each tissue specimen, and two random, non-overlapping fields per slide were obtained. Immediately after removal, heart and liver tissues were fixed in $10 \%$ neutral buffered formalin for $3 \mathrm{~d}$ and then dehydrated and embedded in paraffin wax ${ }^{(14,24)}$. Thin sections $(5 \mu \mathrm{m})$ of the left ventricle and the liver were cut and stained with haematoxylineosin stain for determining inflammatory cell infiltration with $20 \times$ and fat vacuole enlargement with $20 \times$ objectives using an Olympus BX51 microscope (Olympus). Collagen distribution was evaluated in the left ventricle with picrosirius red stain. Laser confocal microscopy (Zeiss LSM 510 upright Confocal Microscope; Carl Zeiss) with colour intensity quantified using NIH-imageJ software (National Institute of Health) was used to determine the extent of collagen deposition in the selected tissue sections $^{(14,24)}$. Faecal matter-free ileal tissue segments were removed from the mesenteric border by rinsing and fixed in $10 \%$ neutral buffered formalin for $3 \mathrm{~d}$, dehydrated and embedded in paraffin wax ${ }^{(14,24)}$. The ileal segments were cut at 5 - $\mu \mathrm{m}$ thickness and stained with haematoxylin-eosin for determining inflammatory cell infiltration, mucosal thickening, gland (crypts) cell proliferation and morphology with $20 \times$ objective using an Olympus BX51 microscope (Olympus) ${ }^{(25,26)}$.

\section{Plasma analyses}

Blood samples were centrifuged at $5000 \boldsymbol{g}$ for $15 \mathrm{~min}$ within $30 \mathrm{~min}$ of collection into heparinised tubes. Plasma samples were separated and transferred to Eppendorf tubes for storage at $-20^{\circ} \mathrm{C}$ before analysis. Activities of plasma liver enzymes, alanine transaminase (ALT) and aspartate transaminase (AST), and concentrations of plasma analytes including NEFA, TAG and total cholesterol were determined using kits and controls supplied by Olympus using an Olympus analyser $(\mathrm{AU} 400)^{(14,24)}$.

\section{Inulin oligofructose mixture}

Orafti Synergy1, an oligofructose-enriched inulin mixture ${ }^{(15)}$, supplied by Invita Australia Pty Ltd, contained 92 (SEM 2) g of oligofructose + inulin, 8 (SEM 2) g of sugars (glucose + fructose + sucrose) and sulphated ash content $<0.2 \mathrm{~g} / 100 \mathrm{~g}$. The energy value of Synergy 1 mixture was $693 \mathrm{~kJ} / 100 \mathrm{~g}$ of powdered mixture.

\section{Statistical analysis}

All data are presented as means with their standard errors. Results were tested for variance using Bartlett's test, and variables that were not normally distributed were transformed (using $\log 10$ function) before statistical analyses. Data from C, $\mathrm{CIO}, \mathrm{H}$ and $\mathrm{HIO}$ rats were tested by two-way ANOVA. When interaction and/or the main effects were significant, means were compared using Newman-Keuls multiple comparison post hoc test. Where transformations did not result in normality or constant variance, a Kruskal-Wallis non-parametric test was performed. A $P$ value of $<0.05$ was considered as statistically significant. All statistical analyses were performed using GraphPad Prism version 5.00 for Windows.

\section{Results}

Inulin oligofructose treatment on food intake, body weight, plasma lipid profile and fat mass

Feeding a high-energy diet together with fructosesupplemented drinking water reduced food and water intake in $\mathrm{H}$ and $\mathrm{HIO}$ rats compared with $\mathrm{C}$ and $\mathrm{CIO}$ rats (Table 1). IO in $\mathrm{CIO}$ and $\mathrm{HIO}$ rats did not change food or water intake, compared with the respective $\mathrm{C}$ and $\mathrm{H}$ rats (Table 1). With increased energy in the diet, $\mathrm{H}$ rats increased energy intake with a corresponding increase in feed conversion efficiency and body weight gain compared with $\mathrm{C}$ and $\mathrm{CIO}$ rats (Table 1 ). IO did not change energy intake in HIO rats, but increased energy intake in $\mathrm{CIO}$ rats compared with $\mathrm{C}$ rats (Table 1). IO attenuated body weight gain with reduced feed conversion efficiency in $\mathrm{CIO}$ or HIO rats (Table 1).

The high-energy diet with increased simple carbohydrates and SFA increased the plasma concentrations of TAG, NEFA and total cholesterol in $\mathrm{H}$ rats compared with $\mathrm{C}$ rats (Table 1). IO improved the lipid profile with reduced plasma concentration of TAG and NEFA in HIO rats, whereas in CIO rats plasma NEFA concentrations increased compared with $\mathrm{C}$ rats (Table 1). 
Table 1. Dietary intakes, plasma lipid profile and lipid output

(Mean values with their standard errors; eight to ten rats per group)

\begin{tabular}{|c|c|c|c|c|c|c|c|c|c|c|c|}
\hline \multirow[b]{2}{*}{ Variables } & \multicolumn{2}{|c|}{ C } & \multicolumn{2}{|c|}{$\mathrm{ClO}$} & \multicolumn{2}{|c|}{$\mathrm{H}$} & \multicolumn{2}{|c|}{$\mathrm{HIO}$} & \multicolumn{3}{|c|}{$P$} \\
\hline & Mean & SEM & Mean & SEM & Mean & SEM & Mean & SEM & Diet & Treatment & Interaction \\
\hline Food intake $(\mathrm{g} / \mathrm{d})$ & $31 \cdot 8^{a}$ & 1.7 & $33 \cdot 0^{\mathrm{a}}$ & $2 \cdot 2$ & $20 \cdot 9^{b}$ & 1.4 & $20 \cdot 8^{\mathrm{b}}$ & 1.2 & $<0.0001$ & 0.73 & 0.68 \\
\hline Water intake (ml/d) & $31 \cdot 2^{\mathrm{a}}$ & 1.7 & $32 \cdot 7^{\mathrm{a}}$ & $2 \cdot 1$ & $18 \cdot 7^{\mathrm{b}}$ & 1.5 & $19 \cdot 3^{\mathrm{b}}$ & 1.5 & $<0.0001$ & 0.54 & 0.78 \\
\hline Energy intake $(\mathrm{kJ} / \mathrm{d})$ & $362.4^{c}$ & 3.6 & $389 \cdot 3^{\mathrm{b}}$ & $5 \cdot 5$ & $438.6^{\mathrm{a}}$ & $5 \cdot 7$ & $446 \cdot 6^{\mathrm{a}}$ & 5.5 & $<0.0001$ & 0.0017 & 0.07 \\
\hline Feed conversion efficiency (\%) & $2 \cdot 4^{\mathrm{b}}$ & 0.1 & $0.9^{c}$ & 0.5 & $4 \cdot 6^{\mathrm{a}}$ & 0.3 & $1.8^{\mathrm{b}}$ & 0.1 & $<0.0001$ & $<0.0001$ & 0.09 \\
\hline Body weight gain $(\%)^{*}$ & $8.7^{\mathrm{b}}$ & 0.3 & $3.4^{c}$ & $2 \cdot 1$ & $20 \cdot 2^{a}$ & 1.3 & $7.9^{\mathrm{b}}$ & 0.6 & $<0.0001$ & $<0.0001$ & 0.0155 \\
\hline Plasma NEFA (mmol/l) & $1.65^{\mathrm{c}}$ & 0.27 & $2 \cdot 93^{b}$ & 0.47 & $5 \cdot 12^{\mathrm{a}}$ & 0.33 & $3.78^{b}$ & 0.51 & $<0.0001$ & 0.94 & 0.003 \\
\hline Plasma TAG (mmol/l) & $0.49^{\mathrm{b}}$ & 0.07 & $0.54^{\mathrm{b}}$ & 0.09 & $2.69^{a}$ & 0.43 & $0.65^{b}$ & 0.11 & $<0.0001$ & 0.0002 & $<0.0001$ \\
\hline Plasma total cholesterol $(\mathrm{mmol} / \mathrm{l})$ & $1.54^{\mathrm{b}}$ & 0.06 & $1.58^{\mathrm{b}}$ & 0.09 & $1.99^{a}$ & 0.04 & $1.88^{\mathrm{a}}$ & 0.08 & $<0.0001$ & 0.62 & 0.29 \\
\hline Faecal lipid excretion (\%) & $7.37^{\mathrm{c}}$ & 0.97 & $8.92^{\mathrm{c}}$ & 0.45 & $14 \cdot 37^{b}$ & 0.61 & $19 \cdot 81^{\mathrm{a}}$ & 1.52 & $<0.0001$ & 0.002 & 0.06 \\
\hline
\end{tabular}

$\mathrm{C}$, corn starch; $\mathrm{ClO}$, corn starch + inulin oligofructose; $\mathrm{H}$, high carbohydrate, high fat; $\mathrm{HIO}$, high carbohydrate, high fat + inulin oligofructose.

$\mathrm{a}, \mathrm{b}, \mathrm{c}$ Mean values within a row with unlike superscript letters were significantly different $(P<0.05)$.

* Body weight gain percentage calculated as percentage of body weight increased from 8 to 16 weeks for all groups.

Table 2. Fat mass development ${ }^{*}$

(Mean values with their standard errors; eight to ten rats per group)

\begin{tabular}{|c|c|c|c|c|c|c|c|c|c|c|c|}
\hline \multirow[b]{2}{*}{ Variables } & \multicolumn{2}{|c|}{ C } & \multicolumn{2}{|c|}{$\mathrm{ClO}$} & \multicolumn{2}{|c|}{$\mathrm{H}$} & \multicolumn{2}{|c|}{ HIO } & \multicolumn{3}{|c|}{$P$} \\
\hline & Mean & SEM & Mean & SEM & Mean & SEM & Mean & SEM & Diet & Treatment & Interaction \\
\hline Total fat mass (g) & $95 \cdot 2^{b, c}$ & 4.9 & $69 \cdot 6^{\mathrm{c}}$ & $6 \cdot 7$ & $210 \cdot 1^{\mathrm{a}}$ & 14.9 & $121 \cdot 5^{\mathrm{b}}$ & $7 \cdot 3$ & $<0.0001$ & $<0.0001$ & 0.0021 \\
\hline Total lean mass $(\mathrm{g})$ & 279.0 & $2 \cdot 7$ & 299.9 & 7.9 & $279 \cdot 1$ & $10 \cdot 9$ & 308.5 & 7.4 & 0.581 & 0.0032 & 0.590 \\
\hline Abdominal circumference $(\mathrm{cm})$ & $18 \cdot 2^{\mathrm{c}}$ & 0.2 & $17 \cdot 9^{\mathrm{c}}$ & 0.2 & $21.9^{a}$ & 0.3 & $19 \cdot 4^{\mathrm{b}}$ & 0.2 & $<0.0001$ & $<0.0001$ & $<0.0001$ \\
\hline Retroperitoneal fat $(\mathrm{mg} / \mathrm{mm})$ & $122 \cdot 6^{\mathrm{c}}$ & 9.8 & $117 \cdot 5^{\mathrm{c}}$ & $20 \cdot 8$ & $359.5^{a}$ & 33.9 & $195 \cdot 4^{\mathrm{b}}$ & $16 \cdot 4$ & $<0.0001$ & 0.0005 & 0.0009 \\
\hline Epididymal fat $(\mathrm{mg} / \mathrm{mm})$ & $95 \cdot 8^{\mathrm{c}}$ & 6.0 & $93.4^{\mathrm{c}}$ & 13.8 & $212 \cdot 1^{a}$ & $15 \cdot 4$ & $139 \cdot 2^{b}$ & 11.6 & $<0.0001$ & 0.0040 & 0.0066 \\
\hline Omental fat $(\mathrm{mg} / \mathrm{mm})$ & $56 \cdot 8^{\mathrm{C}}$ & 4.5 & $53 \cdot 4^{\mathrm{C}}$ & $6 \cdot 2$ & $116 \cdot 5^{\mathrm{a}}$ & $7 \cdot 7$ & $84 \cdot 3^{b}$ & 6.9 & $<0.0001$ & 0.0089 & 0.0315 \\
\hline Total abdominal fat $(\mathrm{mg} / \mathrm{mm})$ & $275 \cdot 1^{\mathrm{c}}$ & $18 \cdot 9$ & $264 \cdot 3^{\mathrm{c}}$ & $40 \cdot 1$ & $688 \cdot 0^{\mathrm{a}}$ & 54.5 & $418 \cdot 9^{b}$ & $32 \cdot 3$ & $<0.0001$ & 0.0009 & 0.0020 \\
\hline Visceral adiposity index (\%) & $3.3^{\mathrm{c}}$ & 0.2 & $3 \cdot 2^{\mathrm{c}}$ & 0.4 & $6 \cdot 5^{\mathrm{a}}$ & 0.5 & $4 \cdot 7^{\mathrm{b}}$ & 0.4 & $<0.0001$ & 0.0201 & 0.0361 \\
\hline
\end{tabular}

$\mathrm{C}$, corn starch; $\mathrm{ClO}$, corn starch + inulin oligofructose; $\mathrm{H}$, high carbohydrate, high fat; $\mathrm{HIO}$, high carbohydrate, high fat + inulin oligofructose.

$\mathrm{a}, \mathrm{b}, \mathrm{c}$ Mean values within a row with unlike superscript letters were significantly different $(P<0.05)$.

* Body weight gain percentage calculated as percentage of body weight increased from 8 to 16 weeks for all groups.

However, plasma cholesterol concentrations remained unchanged in $\mathrm{CIO}$ and $\mathrm{HIO}$ rats compared with $\mathrm{C}$ and $\mathrm{H}$ rats (Table 1).

$\mathrm{H}$ rats had increased total body fat, abdominal fat mass and abdominal circumference compared with $\mathrm{C}$ rats (Table 2). IO reduced abdominal (39\%) and total body fat mass (42\%) associated with reduced abdominal circumference (11\%) in HIO rats (Table 2$)$. IO reduced total body fat mass $(27 \%)$ in CIO compared with $\mathrm{C}$ rats without changing abdominal circumference (Table 2).

\section{Inulin oligofructose treatment on gut morphology and function and faecal output}

$\mathrm{H}$ rats had increased weight of stomach, small intestine, caecum and colon with contents as well as total weight compared with $\mathrm{C}$ rats (Table 3 ). $\mathrm{H}$ rats showed elongated ileal crypt cells with a reduced density and an increased intestinal ileal inflammation compared with $\mathrm{C}$ rats (Fig. 1(a) and (c)). IO increased caecum and colon weights in $\mathrm{CIO}$ and $\mathrm{HIO}$ rats (Table 3). Stool production increased by 1.9 or $1.7 \mathrm{~g} / 12 \mathrm{~h}$ in $\mathrm{CIO}$ and $\mathrm{HIO}$ rats compared with $\mathrm{C}$ and $\mathrm{H}$ rats (Table 3 ). IO improved gut morphology with reduced intestinal ileal inflammation, enhanced density of crypt cells with no signs of elongation and improved villi morphology in HIO rats compared with $\mathrm{H}$ rats
(Fig. 1(c) and (d)). Furthermore, no changes were observed in the length of gastrointestinal segments (Table 3).

\section{Inulin oligofructose treatment on liver structure and function and glucose and insulin responses}

H-rats showed increased liver weights (Table 4) with enhanced fat accumulation and infiltration of inflammatory cells (Fig. 2(a), (c), (e) and (g)) as well as increased plasma activities of the liver enzymes, ALT and AST, compared with C rats (Table 4). IO decreased liver weight by $16.7 \%$ and reversed all other liver changes in HIO rats (Table 4; Fig. 2(c), (d), (g) and (h)).

Hyperglycaemia, impaired oral glucose tolerance and insulin resistance were measured in $\mathrm{H}$ rats compared with $\mathrm{C}$ and $\mathrm{CIO}$ rats (Table 4). IO improved the blood glucose profile with reduced fasting blood glucose concentrations associated with improved oral glucose tolerance and insulin tolerance in HIO rats compared with $\mathrm{H}$ rats (Table 4).

\section{Inulin oligofructose treatment on cardiovascular structure and function}

$\mathrm{H}$ rats showed increased SBP (Table 5) as well as decreased endothelium-dependent relaxation to acetylcholine (Fig. 3(C)) 
Table 3. Gastrointestinal weight and faecal output*

(Mean values with their standard errors; ten rats per group)

\begin{tabular}{|c|c|c|c|c|c|c|c|c|c|c|c|}
\hline \multirow[b]{2}{*}{ Variables } & \multicolumn{2}{|c|}{ C } & \multicolumn{2}{|c|}{$\mathrm{ClO}$} & \multicolumn{2}{|c|}{$\mathrm{H}$} & \multicolumn{2}{|c|}{$\mathrm{HIO}$} & \multicolumn{3}{|c|}{$P$} \\
\hline & Mean & SEM & Mean & SEM & Mean & SEM & Mean & SEM & Diet & Treatment & Interaction \\
\hline Stomach (mg/mm) & $80 \cdot 4^{\mathrm{b}}$ & 9.5 & $89.0^{\mathrm{b}}$ & 8.6 & $133 \cdot 2^{\mathrm{a}}$ & $12 \cdot 9$ & $118 \cdot 9^{\mathrm{a}, \mathrm{b}}$ & $13 \cdot 7$ & 0.052 & 0.566 & 0.132 \\
\hline Small intestine $(\mathrm{cm})$ & 143.5 & $2 \cdot 2$ & $139 \cdot 7$ & 4.4 & $148 \cdot 8$ & 1.8 & $142 \cdot 2$ & 1.7 & 0.165 & 0.067 & 0.614 \\
\hline Proximal colon $(\mathrm{cm})$ & 4.7 & $0 \cdot 1$ & $5 \cdot 7$ & 0.3 & 4.7 & 0.3 & 5.8 & 0.4 & 0.867 & 0.0011 & 0.867 \\
\hline Distal colon $(\mathrm{cm})$ & $3.7^{\mathrm{a}}$ & 0.1 & $3 \cdot 5^{a, b}$ & 0.3 & $3 \cdot 0^{\mathrm{b}}$ & 0.1 & $3 \cdot 3^{a, b}$ & 0.1 & 0.0135 & 0.775 & 0.158 \\
\hline Total colon $(\mathrm{cm})$ & 8.3 & 0.2 & 9.2 & 0.5 & 7.8 & 0.3 & 9.1 & 0.4 & 0.420 & 0.005 & 0.590 \\
\hline Small intestine $(\mathrm{mg} / \mathrm{mm})$ & $216 \cdot 7^{b}$ & $9 \cdot 8$ & $209 \cdot 9^{b}$ & 18.5 & $260 \cdot 3^{a}$ & $5 \cdot 3$ & $231 \cdot 2^{a, b}$ & 8.3 & 0.0081 & 0.130 & 0.342 \\
\hline Caecum $(\mathrm{mg} / \mathrm{mm})$ & $80.9^{c}$ & 5.5 & $105 \cdot 6^{\mathrm{b}}$ & 4.0 & $101 \cdot 1^{\mathrm{b}}$ & 5.5 & $124 \cdot 8^{\mathrm{a}}$ & 5.0 & 0.0032 & 0.0003 & 0.497 \\
\hline Colon $(\mathrm{mg} / \mathrm{mm})$ & $30 \cdot 3^{c}$ & 1.9 & $53 \cdot 3^{\mathrm{a}}$ & $3 \cdot 2$ & $40 \cdot 2^{\mathrm{b}}$ & 3.5 & $56.9^{\mathrm{a}}$ & 2.9 & 0.0275 & $<0.0001$ & 0.2907 \\
\hline Total gut (mg/mm) & $408 \cdot 3^{\mathrm{b}}$ & $21 \cdot 7$ & $457.8^{b}$ & $24 \cdot 3$ & $534 \cdot 9^{\mathrm{a}}$ & $18 \cdot 2$ & $525 \cdot 6^{\mathrm{a}}$ & $18 \cdot 9$ & $<0.0001$ & 0.343 & 0.168 \\
\hline Faecal weight $(\mathrm{g} / 12 \mathrm{~h})$ & $1.08^{d}$ & 0.14 & $2.95^{\mathrm{b}}$ & 0.15 & $1.77^{\mathrm{c}}$ & 0.11 & $3.42^{\mathrm{a}}$ & 0.19 & 0.001 & $<0.0001$ & 0.47 \\
\hline
\end{tabular}

$\mathrm{C}$, corn starch; $\mathrm{CIO}$, corn starch + inulin oligofructose; $\mathrm{H}$, high carbohydrate, high fat; $\mathrm{HIO}$, high carbohydrate, high fat + inulin oligofructose

a,b,c,d Mean values within a row with unlike superscript letters were significantly different $(P<0.05)$

* Tissue wet weights were normalised with the tibial length $(\mathrm{mg} / \mathrm{mm})$.
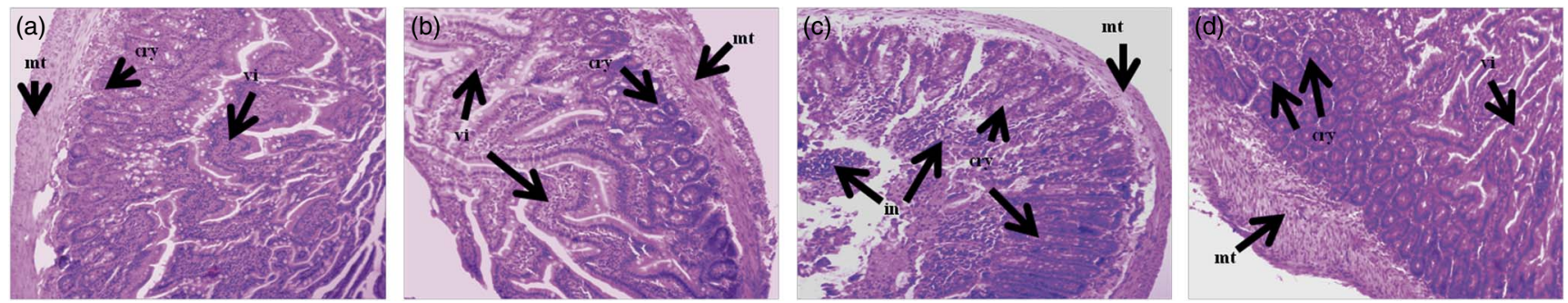

Fig. 1. Haematoxylin-eosin staining of the ileum ( $\times 20)$ showing inflammatory cells infiltration (a-d), with inflammatory cells marked as 'in', crypt cells (a-d) marked as 'cry', villi (a-d) marked as 'vi' and mucosal thickening (a-d) marked as ' $m$ t' in rats fed corn starch diet (a), corn starch diet + inulin oligofructose (b), high-carbohydrate, high-fat diet (c), high-carbohydrate, high-fat diet + inulin oligofructose (d).

Table 4. Hepatic structure and function and glycaemic profile

(Mean values with their standard errors; eight to ten rats per group)

\begin{tabular}{|c|c|c|c|c|c|c|c|c|c|c|c|}
\hline \multirow[b]{2}{*}{ Variables } & \multicolumn{2}{|c|}{$\mathrm{C}$} & \multicolumn{2}{|c|}{$\mathrm{ClO}$} & \multicolumn{2}{|c|}{$\mathrm{H}$} & \multicolumn{2}{|c|}{$\mathrm{HIO}$} & \multicolumn{3}{|c|}{$P$} \\
\hline & Mean & SEM & Mean & SEM & Mean & SEM & Mean & SEM & Diet & Treatment & Interaction \\
\hline Liver weight $(\mathrm{mg} / \mathrm{mm})$ & $243.5^{\mathrm{c}}$ & 4.9 & $260 \cdot 7^{\mathrm{b}, \mathrm{c}}$ & $11 \cdot 2$ & $342 \cdot 6^{\mathrm{a}}$ & 11.7 & $285 \cdot 3^{\mathrm{b}}$ & $10 \cdot 3$ & $<0.0001$ & 0.050 & 0.0006 \\
\hline Plasma ALT activity (U/l) & $29 \cdot 6^{\mathrm{b}}$ & $2 \cdot 8$ & $32 \cdot 1^{\mathrm{b}}$ & $2 \cdot 9$ & $43 \cdot 9^{\mathrm{a}}$ & $2 \cdot 6$ & $33 \cdot 6^{\mathrm{b}}$ & $2 \cdot 0$ & 0.005 & 0.14 & 0.019 \\
\hline Plasma AST activity (U/I) & $74.4^{\mathrm{b}}$ & $3 \cdot 2$ & $74.9^{\mathrm{b}}$ & $4 \cdot 2$ & $96 \cdot 8^{\mathrm{a}}$ & $7 \cdot 2$ & $70 \cdot 6^{\mathrm{b}}$ & 3.2 & 0.07 & 0.011 & 0.009 \\
\hline Fasting blood glucose concentration (mmol/l) & $4 \cdot 5^{\mathrm{b}}$ & 0.1 & $4 \cdot 2^{b}$ & 0.1 & $5 \cdot 2^{\mathrm{a}}$ & 0.1 & $4 \cdot 3^{\mathrm{b}}$ & 0.1 & 0.0003 & $<0.0001$ & 0.005 \\
\hline \multicolumn{12}{|l|}{ OGTT (AUC) $(\mathrm{mmol} / \mathrm{l}$ per $\mathrm{min})$} \\
\hline Week 0 & 640.5 & $20 \cdot 6$ & 633.5 & $15 \cdot 6$ & $610 \cdot 5$ & $25 \cdot 1$ & $629 \cdot 6$ & $17 \cdot 1$ & 0.40 & 0.76 & 0.52 \\
\hline Week 8 & $654.8^{\mathrm{b}}$ & $9 \cdot 6$ & $660 \cdot 0^{\mathrm{b}}$ & 14.5 & $826 \cdot 1^{\mathrm{a}}$ & 6.9 & $845 \cdot 1^{\mathrm{a}}$ & 11.4 & $<0.0001$ & 0.277 & 0.533 \\
\hline Week 16 & $665 \cdot 6^{\mathrm{C}}$ & $14 \cdot 7$ & $653.4^{\mathrm{C}}$ & $10 \cdot 6$ & $838 \cdot 7^{\mathrm{a}}$ & $20 \cdot 3$ & $728 \cdot 7^{b}$ & 13.8 & $<0.0001$ & 0.0004 & 0.0025 \\
\hline ITT (AUC) (mmol// per min) & $187 \cdot 4^{\mathrm{c}}$ & 24.4 & $155 \cdot 2^{c}$ & 11.4 & $531 \cdot 0^{\mathrm{a}}$ & 18.5 & $323.9^{b}$ & 11.0 & $<0.0001$ & $<0.0001$ & $<0.0001$ \\
\hline
\end{tabular}

$\mathrm{C}$, corn starch; $\mathrm{CIO}$, corn starch + inulin oligofructose; $\mathrm{H}$, high carbohydrate, high fat; HIO, high carbohydrate, high fat + inulin oligofructose; ALT, alanine transaminase; AST, aspartate transaminase; OGTT, oral glucose tolerance; ITT, insulin tolerance.

a,b,c Mean values within a row with unlike superscript letters were significantly different $(P<0.05)$.

and endothelium-independent relaxation to sodium nitroprusside (Fig. 3(B)) compared with $\mathrm{C}$ and $\mathrm{CIO}$ rats (Table 5). IO decreased SBP (Table 5) and increased vascular relaxant responses to both acetylcholine and sodium nitroprusside, compared with $\mathrm{H}$ rats (Fig. 3(C) and (B)). Using echocardiographic examination, most of the cardiovascular parameters remained unchanged, but increased SBP:LVIDs and ESS:LVIDs ratios demonstrated that increased LV contractility was observed in $\mathrm{H}$ rats compared with $\mathrm{C}$ rats (Table 5). IO treatment may improve LV contractility with the normalised SBP:LVIDs and ESS:LVIDs ratios in HIO rats, compared with the $\mathrm{H}$ rats (Table 5). The improvement in LV contractility with IO treatment did not change either heart weight or left ventricle+septum weight measurements in HIO rats (Table 5). LV diastolic stiffness was increased in $\mathrm{H}$ rats and normalised in HIO rats (Table 5). H rats showed increased infiltration of inflammatory cells accompanied by enhanced collagen deposition in the left ventricle compared with $\mathrm{C}$ and $\mathrm{CIO}$ rats (Fig. 4(a), (b), (c), (e), (f) and (g)). Minimal inflammatory cell infiltration and markedly reduced collagen deposition were observed in HIO rats (Fig. 4(c), (d), (g) and (h)). 

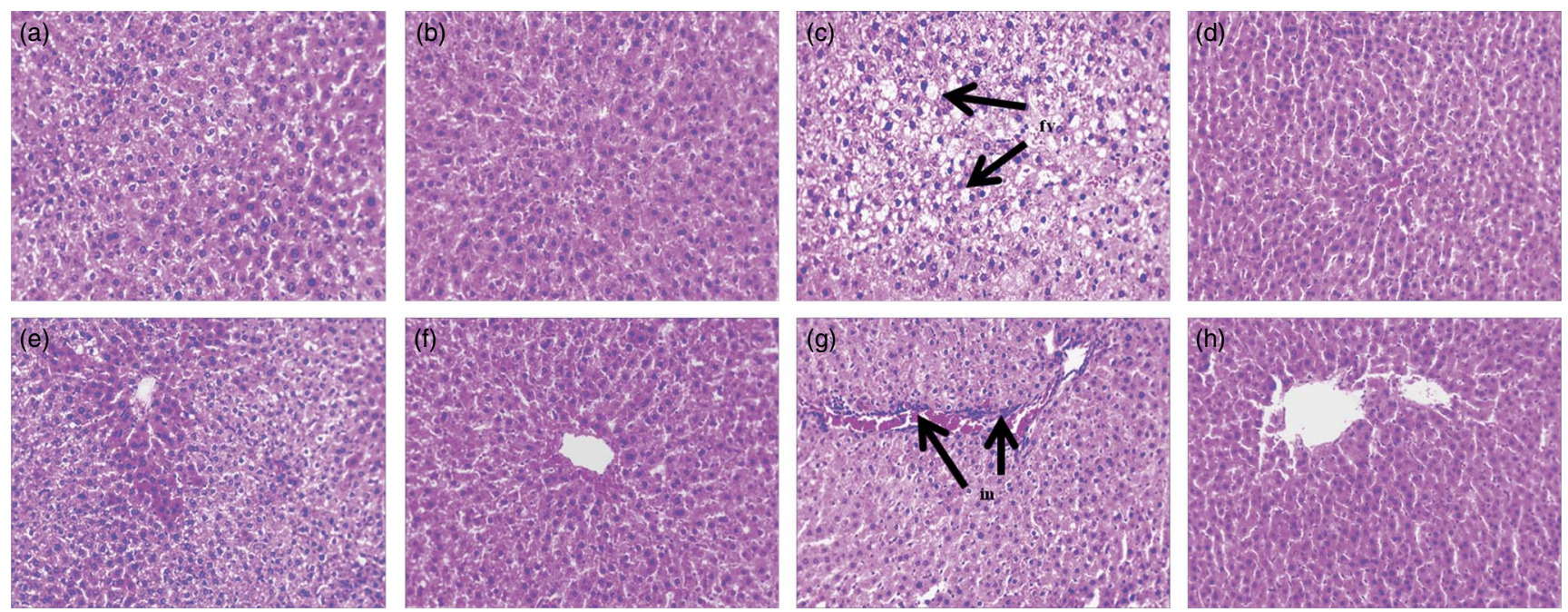

Fig. 2. Haematoxylin-eosin staining of hepatocytes $(\times 20)$ showing hepatocytes with enlarged fat vacuoles ((a-d), fat vacuoles marked as 'fv') and inflammatory cells infiltration ((e-h), inflammatory cells marked as 'in') $(20 x)$ in rats fed corn starch diet $(a, e)$, corn starch diet + inulin oligofructose (b, f), high-carbohydrate, high-fat diet $(c, g)$, high-carbohydrate, high-fat diet + inulin oligofructose $(d, h)$.

Table 5. Cardiovascular structure and function

(Mean values with their standard errors)

\begin{tabular}{|c|c|c|c|c|c|c|c|c|c|c|c|}
\hline \multirow[b]{2}{*}{ Variables } & \multicolumn{2}{|c|}{ C } & \multicolumn{2}{|c|}{$\mathrm{ClO}$} & \multicolumn{2}{|c|}{$\mathrm{H}$} & \multicolumn{2}{|c|}{$\mathrm{HIO}$} & \multicolumn{3}{|c|}{$P$} \\
\hline & Mean & SEM & Mean & SEM & Mean & SEM & Mean & SEM & Diet & Treatment & Interaction \\
\hline $\mathrm{SBP}(\mathrm{mmHg})$ & $124^{\mathrm{b}}$ & 2 & $118^{\mathrm{b}}$ & 2 & $152^{\mathrm{a}}$ & 2 & $123^{b}$ & 3 & $<0.0001$ & $<0.0001$ & $<0.0001$ \\
\hline Heart rate (beats per min) & $308 \cdot 0^{\mathrm{a}}$ & $24 \cdot 1$ & $237.0^{\mathrm{b}}$ & $9 \cdot 0$ & $301 \cdot 3^{a}$ & $17 \cdot 2$ & $259 \cdot 9^{a, b}$ & $12 \cdot 3$ & 0.617 & 0.0014 & 0.363 \\
\hline LVIDd (mm) & 7.73 & 0.22 & $7 \cdot 71$ & 0.12 & 7.87 & 0.18 & 7.95 & $0 \cdot 18$ & 0.295 & 0.868 & 0.781 \\
\hline LVIDs (mm) & 4.40 & 0.22 & $4 \cdot 10$ & 0.22 & $4 \cdot 17$ & 0.21 & 4.42 & 0.24 & 0.841 & 0.911 & 0.227 \\
\hline IVSd (mm) & 1.92 & 0.07 & 1.88 & 0.03 & 2.03 & 0.04 & 1.90 & 0.02 & 0.152 & 0.065 & 0.317 \\
\hline IVSs (mm) & 3.07 & 0.13 & 3.17 & 0.10 & 3.19 & 0.16 & $2 \cdot 89$ & $0 \cdot 10$ & 0.527 & 0.430 & 0.121 \\
\hline LVPWd $(\mathrm{mm})$ & 1.79 & 0.11 & 1.88 & 0.03 & 1.99 & 0.04 & 1.83 & 0.03 & 0.238 & 0.578 & 0.054 \\
\hline LVPWs (mm) & 2.79 & 0.07 & 2.99 & 0.28 & 2.96 & 0.11 & 2.95 & $0 \cdot 11$ & 0.695 & 0.567 & 0.527 \\
\hline Relative wall thickness & 0.48 & 0.02 & 0.49 & 0.01 & 0.51 & 0.01 & 0.47 & 0.01 & 0.708 & 0.267 & 0.069 \\
\hline Systolic wall stress & $97 \cdot 2$ & 5.8 & $85 \cdot 2$ & 9.4 & $107 \cdot 2$ & 8.0 & 91.6 & 5.5 & 0.274 & 0.071 & 0.808 \\
\hline Diastolic volume $(\mu \mathrm{l})$ & 493.0 & $39 \cdot 2$ & $481 \cdot 0$ & $22 \cdot 4$ & $517 \cdot 0$ & 34.7 & $532 \cdot 0$ & 35.9 & 0.275 & 0.965 & 0.691 \\
\hline Systolic volume $(\mu \mathrm{l})$ & 94.0 & $13 \cdot 3$ & $77 \cdot 0$ & $13 \cdot 3$ & $80 \cdot 0$ & 12.4 & 96.0 & $15 \cdot 5$ & 0.856 & 0.971 & 0.238 \\
\hline Stroke volume $(\mu \mathrm{l})$ & 399.0 & 31.3 & 404.0 & $17 \cdot 7$ & 437.0 & $29 \cdot 3$ & 436.0 & $30 \cdot 1$ & 0.216 & 0.943 & 0.914 \\
\hline Cardiac output (ml/min) & $118 \cdot 2$ & $5 \cdot 2$ & $96 \cdot 2$ & 6.4 & 130.4 & $9 \cdot 8$ & $115 \cdot 2$ & 13.7 & $0 \cdot 107$ & 0.057 & 0.720 \\
\hline Fractional shortening (\%) & $45 \cdot 0$ & 3.0 & $49 \cdot 3$ & $1 \cdot 1$ & $47 \cdot 2$ & $2 \cdot 1$ & 46.5 & 1.7 & 0.887 & 0.397 & 0.242 \\
\hline Ejection fraction (\%) & $81 \cdot 1$ & 1.9 & $84 \cdot 3$ & $2 \cdot 2$ & 84.8 & 1.8 & $82 \cdot 2$ & $2 \cdot 2$ & 0.697 & 0.884 & 0.165 \\
\hline SBP:LVIDs & $284^{\mathrm{b}}$ & 16 & $291^{\mathrm{b}}$ & 15 & $367^{\mathrm{a}}$ & 19 & $281^{\mathrm{b}}$ & 18 & 0.044 & 0.033 & 0.013 \\
\hline SBP:systolic volume & 1538 & 252 & 1807 & 263 & 2192 & 293 & 1524 & 252 & 0.491 & 0.458 & 0.089 \\
\hline ESS:LVIDs & $221^{\mathrm{b}}$ & 8 & $206^{\mathrm{b}}$ & 16 & $256^{\mathrm{a}}$ & 10 & $208^{\mathrm{b}}$ & 8 & $0 \cdot 10$ & 0.007 & $0 \cdot 14$ \\
\hline Diastolic stiffness $(\kappa)$ & $22 \cdot 9^{b}$ & 0.6 & $22 \cdot 9^{\mathrm{b}}$ & 0.5 & $27 \cdot 8^{\mathrm{a}}$ & 0.5 & $22 \cdot 6^{\mathrm{b}}$ & 1.2 & 0.0045 & 0.0015 & 0.0015 \\
\hline Estimated LV mass $(\mathrm{g})$ & 1.09 & 0.09 & 1.09 & 0.04 & 1.25 & 0.06 & $1 \cdot 14$ & 0.03 & 0.089 & 0.364 & 0.364 \\
\hline $\mathrm{LV}$ + septum wet weight $(\mathrm{mg} / \mathrm{mm})^{*}$ & $16 \cdot 79^{b}$ & 0.36 & $16 \cdot 21^{\mathrm{b}}$ & 1.06 & $19 \cdot 63^{\mathrm{a}}$ & 0.57 & $17 \cdot 82^{\mathrm{a}, \mathrm{b}}$ & 0.60 & 0.001 & 0.219 & 0.665 \\
\hline RV wet weight $(\mathrm{mg} / \mathrm{mm})^{\star}$ & $2 \cdot 24$ & 0.10 & 2.54 & 0.18 & $2 \cdot 76$ & 0.16 & $2 \cdot 39$ & 0.23 & 0.343 & 0.689 & 0.030 \\
\hline
\end{tabular}

C, corn starch; CIO, corn starch + inulin oligofructose; H, high carbohydrate, high fat; HIO, high carbohydrate, high fat + inulin oligofructose; LVIDd, left ventricular internal diameter thickness in diastole; LVIDs, left ventricular internal diameter thickness in systole; IVSd, interventricular septum thickness in diastole; IVSs, interventricular septum thickness in systole; LVPWd, left ventricular posterior wall thickness in diastole; LVPWs, left ventricular posterior wall thickness in systole; ESS, end-systolic stress; LV, left ventricular; RV, right ventricular.

a,b Mean values within a row with unlike superscript letters were significantly different $(P<0.05)$.

* Tissue wet weights were normalised with the tibial length $(\mathrm{mg} / \mathrm{mm})$.

\section{Discussion}

Intervention with IO in rats fed a high-carbohydrate, high-fat diet improved gastrointestinal structure and function in this study. We propose that these gastrointestinal changes led to improvement in signs of the metabolic syndrome, allowing this non-absorbed prebiotic to be defined as a functional food for this syndrome. We showed that IO did not affect food intake but attenuated weight gain, potentially due to increased faecal loss, as an increase in colonic motility ${ }^{(27)}$ enhanced energy excretion $^{(28)}$. Increased faecal lipid excretion in $\mathrm{H}$ rats treated with IO is indirect evidence for incomplete intestinal fatty acid absorption $^{(27)}$. The total energy expenditure mediated via increased oxidation of endogenous fat may be increased to 
(A)

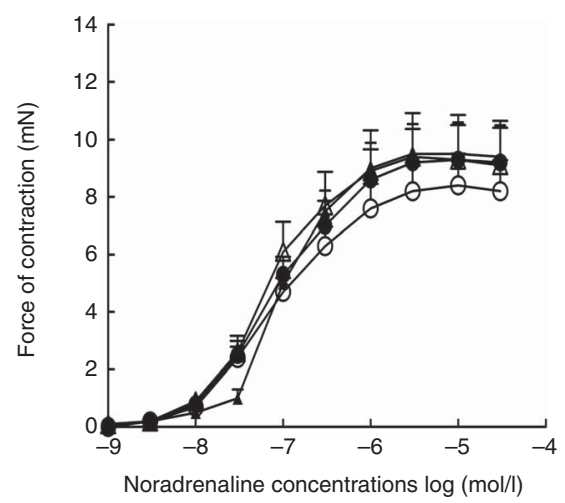

(B) Sodium nitroprusside concentration $\log (\mathrm{mol} / \mathrm{l})$

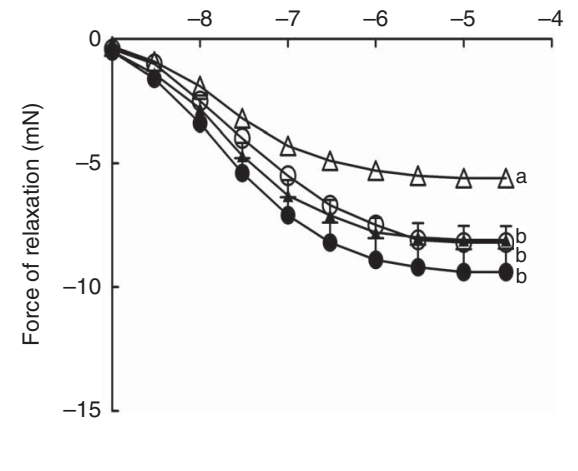

(C) Acetylcholine concentration $\log (\mathrm{mol} / \mathrm{l})$

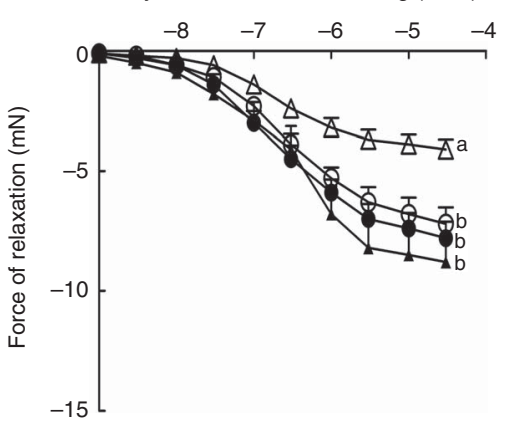

Fig. 3. Cumulative concentration-response curves for noradrenaline (A), sodium nitroprusside (B) and acetylcholine (C) in thoracic aortic rings from rats fed corn starch $\left(\mathrm{C},-\mathrm{O}_{-}\right)$, corn starch + inulin oligofructose $(\mathrm{ClO},--)$, high-carbohydrate, high-fat diet $(\mathrm{H}, \triangle)$ ), high-carbohydrate, high-fat + inulin oligofructose diet ( $\mathrm{HIO}$, - . Values are means, ten rats per group, with their standard errors represented by vertical bars. ${ }^{a, b}$ Mean values with unlike letters are significantly different $(P<0.05)$.

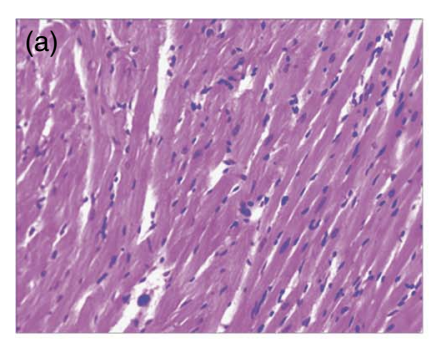

\section{(e)}

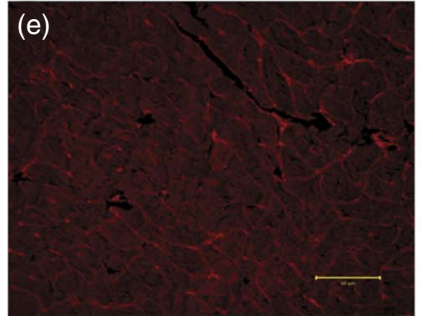

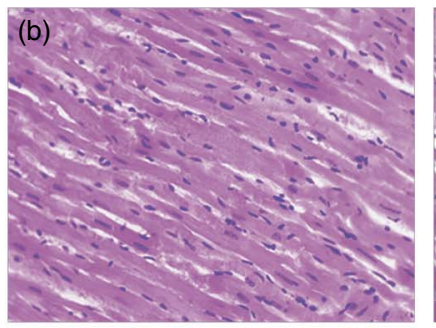

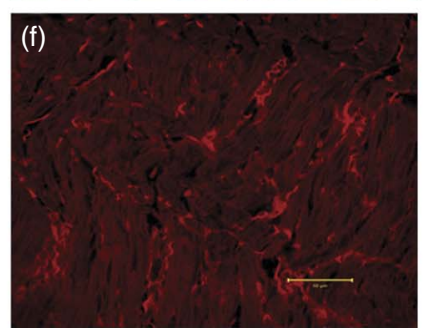

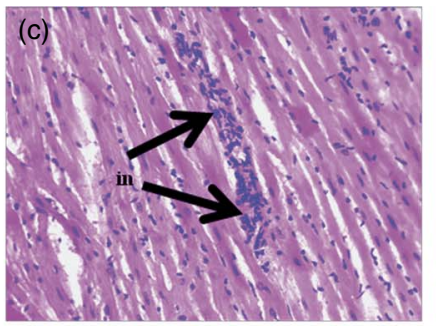

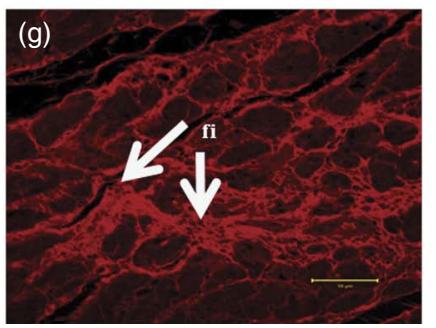

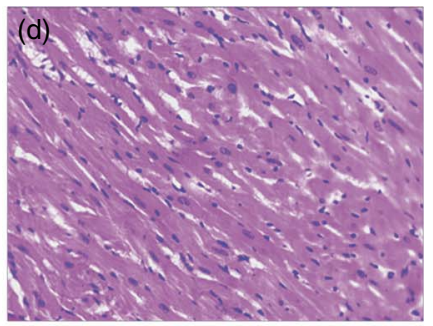

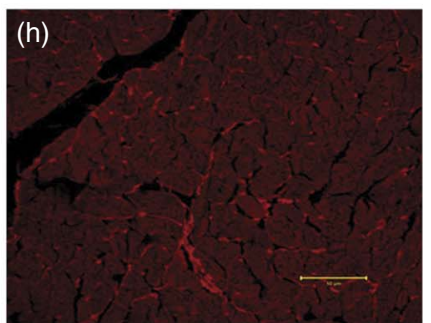

Fig. 4. Haematoxylin-eosin staining of the left ventricle $(\times 20)$ showing infiltration of inflammatory cells ((a-d), inflammatory cells marked as 'in') in rats fed corn starch diet (a), corn starch diet + inulin oligofructose (b), high-carbohydrate, high-fat diet (c), high-carbohydrate, high-fat diet+inulin oligofructose (d). Picrosirius red staining of left ventricular interstitial collagen deposition $((\mathrm{e}-\mathrm{h})$, fibrosis marked as 'fi') $(20 \mathrm{x})$ in rats fed corn starch diet $(\mathrm{e})$, corn starch diet + inulin oligofructose (f), high-carbohydrate, high-fat diet $(\mathrm{g})$, high-carbohydrate, high-fat diet + inulin oligofructose $(\mathrm{h})$.

compensate for this faecal energy loss accompanied by increased faecal lipid excretion ${ }^{(29,30)}$, thus reducing total body fat as well as abdominal fat mass in HIO rats $^{(31)}$ (Table 2). Our results differ from previous studies using rodents where supplementation with $10-20 \%$ inulin and oligofructose in comparison with $5 \%$ in this study prevented weight gain and increased fat mass by reducing food intake via up-regulating the expression of satiety hormones such as glucagon-like peptide-1 and peptide $\mathrm{YY}^{(32-34)}$. Increased doses of inulin as a viscous solution may produce stomach distension by increasing 
retention of water, thereby triggering the vagal responses associated with satiety. The lower dose in our study may improve gastrointestinal motility without stimulation of vagal signals, thus not affecting appetite and food intake ${ }^{(35,36)}$.

Inulin and oligofructose have been reported as effective dietary fibre producing minimal digestion in the stomach but increased fermentation in the colon; these characteristics are likely to provide physiological benefits to metabolically impaired patients, especially for the management of obesity $^{(36-38)}$. Consumption of high-fat diets gradually slows down gastric emptying because of the suppression of gastrointestinal motility ${ }^{(39,40)}$. This is reflected in our study, as high-carbohydrate, high-fat feeding increased stomach weight by $66 \%$ and small intestine weight by $20 \%$ (Table 3). IO also increased caecal weight with contents (19-23\%) (Table 3); this change has been directly linked to the increase in caecal pool size accelerated by colonic fermentation of IO that produces short-chain carboxylic acids such as acetate, butyrate and propionate $^{(41)}$ and decreases caecal $\mathrm{pH}^{(37,42)}$. Further, the fermentation of IO improved intestinal colonic motility ${ }^{(16)}$, shown by increased colon weight with contents and higher stool production $^{(27,37)}$ (Table 3 ) in IO rats. This improvement in gastrointestinal motility with IO is likely to be associated with increased growth of beneficial gut microbes ${ }^{(43)}$. Oral treatment with inulin and oligofructose as a prebiotic mixture promoted the growth of Bifidobacterium and Lactobacillus, with softer stool production likely to improve the innate and adaptive immune responses of younger children ${ }^{(44)}$. Similarly, high dietary fibre treatment that comprised soluble prebiotics including fiberosol-2, fructose-oligosaccharides and oligoisomaltose improved gut ecology with an increased population count of Bifidobacterium pseudocatenulatum in morbidly obese children of both genetic obesity and dietinduced obesity phenotypes ${ }^{(45)}$. This improvement in the gut microbiota profile reversed the dysmicrobiota-mediated toxic metabolite production accompanied by reduced body weight gain and improved metabolic profile in these children ${ }^{(45)}$.

The higher recruitment of lipopolysaccharide (LPS)containing gut microbes ${ }^{(46)}$ expected in $\mathrm{H}$ diet-induced changes in gut flora decreased mucosal ileal wall thickness ${ }^{(46,47)}$ with more inflammation in the intestinal ileum ${ }^{(48,49)}$, as shown in our study. The growth of bifidobacteria should be enhanced by $\mathrm{IO}^{(41)}$ as well as by attenuation of obesity ${ }^{(50,51)}$ and prevention of LPS-induced infiltration of inflammatory cells in the intestinal ileum $^{(64)}$, as in this study. Increased bifidobacteria could attenuate the high-fat diet-induced translocation of LPScontaining gut microbes ${ }^{(52,53)}$ to improve gut-barrier function with reduced intestinal ileal inflammation in HIO rats. Further, increased secretion of gut hormones such as glucagon-like peptide- 2 with IO may lead to improved density of ileal crypt cells and villi morphology ${ }^{(54,55)}$ as in our study.

We also showed that IO improved insulin sensitivity and glucose uptake, likely associated with diminished absorption of ingested carbohydrates with decreased small intestinal transit time ${ }^{(27,37)}$. Further, the likely increase in propionate production and absorption from the colon with IO may reduce plasma concentrations of free fatty acids, and therefore inhibit hepatic glucose production in $\mathrm{HIO}$ rats $^{(56-58)}$. In contrast to our study, neither inulin nor oligofructose produced anti-hyperglycaemic effects in rodents or humans, but the mechanisms of action to improve glucose disposal and insulin sensitivity remain poorly understood $^{(37)}$. The attenuation of total body fat mass accumulation including visceral adiposity development by IO is the likely reason for the improved lipid profile ${ }^{(59)}$ that prevented the incidence of non-alcoholic fatty liver disease with reduced inflammatory cell infiltration of hepatocytes in HIO rats ${ }^{(60-62)}$. IO supplementation in HIO rats improving ileal gut morphology is likely to prevent the release and uptake of bacterial endotoxins such as LPS through a leaky gut barrier and the associated chronic systemic inflammation ${ }^{(63,64)}$; this may precede the adipose tissue inflammation upon $\mathrm{H}$ diet feeding ${ }^{(65)}$. The reduction in endotoxaemia-mediated systemic inflammation $^{(63,66)}$ shown as reduced infiltration of inflammatory cells in the intestinal ileum, heart and liver tissues may attenuate the increased SBP with an improved vascular function in HIO rats $^{(67,68)}$. Concomitantly, an anticipated reduction in leakygut-mediated systemic inflammation, which may be regulated by LPS-activated Toll-like receptor 4 signalling pathway in either parenchyma or bone marrow cells ${ }^{(65,69)}$, may contribute to the reduced LV stiffness accompanied by reduced infiltration of inflammatory cells ${ }^{(66)}$ and collagen deposition in the left ventricle in HIO rats ${ }^{(70,71)}$. Further studies could confirm the LPS-induced systemic inflammation by measuring increased plasma or tissue concentrations of pro-inflammatory cytokines such as monocyte chemo-attractant protein 1 , IL- 6 , interferon- $\gamma$ or TNF- $\alpha^{(69)}$. With this improvement in cardiovascular structure, LV contractility was normalised showing improved cardiovascular structure and function in HIO rats. IO is an effective prebiotic that causes improvements in ileal gut morphology and reduction in intestinal ileal inflammation correlating with improvement in cardiovascular structure and function in $\mathrm{HIO}$ rats.

The Dietitians Association of Australia recommend a daily fibre intake of $25-30 \mathrm{~g}^{(72)}$. The human intake calculated from the intake of IO in HIO rats would be $23.5-37.5 \mathrm{~g} / \mathrm{d}$ based on surface area comparisons ${ }^{(73)}$, meeting these recommendations. As a comparison, metabolic profiles were improved with a marked decrease in body weight by altering the dysbiosis of gut microbiota by administration of $49-51 \mathrm{~g} / \mathrm{d}$ to children with Prader-Willi syndrome or simple obesity ${ }^{(45)}$.

In conclusion, our results indicate that IO treatment contributed to the attenuation of $\mathrm{H}$ diet-induced metabolic complications in HIO rats by improving gut structure and function. IO treatment was associated with reduction in visceral adiposity development; improvement in impaired oral glucose and insulin tolerance, lipid profile, liver structure and function; reduction in infiltration of inflammatory cells into the heart and liver; reduction in SBP with an improved vascular function; and improved cardiovascular structure and function with an improved LV contractility. This model mimics the changes in diet-induced obesity in humans ${ }^{(14)}$ and we have used a dose recommended for humans ${ }^{(67)}$. Thus, our results suggest that this dose of about $30 \mathrm{~g} \mathrm{IO} / \mathrm{d}$ would be sufficient to ameliorate diet-induced metabolic complications in adult humans. Further studies could include analysis of the gut microbiome to determine bacteria that are most affected by chronic IO treatment. 


\section{Acknowledgements}

The authors thank Jason Brightwell (MetroNorth Hospital and Health Service, Brisbane) for the echocardiography measurements in rats.

This study was supported by the University of Southern Queensland Strategic Research Fund (SRF09, L. B. and S. A. K.). The commercial inulin oligofructose mixture (Orafti Synergy1) was provided by Tony Read Shaw, Invita Australia Pty Ltd. The SRF and Invita Australia had no role in the design, analysis or writing of this article.

L. B. and S. A. K. developed the original study aims, interpreted the data and prepared manuscript drafts; S. A. K. conducted the experiments and analysed the data. L. C. W. undertook dual energy X-ray absorptiometry, provided nutritional advice and interpreted the data. L. B. has been the corresponding author throughout the writing process. All authors read and approved the final manuscript.

The authors declare that there are no conflicts of interest.

\section{References}

1. Granado-Lorencio F \& Hernández-Alvarez E (2016) Functional foods and health effects: a nutritional biochemistry perspective. Curr Med Chem (epublication ahead of print version 14 June 2016).

2. Brown L, Poudyal H \& Panchal SK (2015) Functional foods as potential therapeutic options for metabolic syndrome. Obes Rev 16, 914-941.

3. Leach JD \& Sobolik KD (2010) High dietary intake of prebiotic inulin-type fructans in the prehistoric Chihuahuan Desert. Br J Nutr 103, 1558-1561.

4. Florowska A, Krygier K, Florowski T, et al. (2016) Prebiotics as functional food ingredients preventing diet-related diseases. Food Funct 18, 2147-2155.

5. Jones JM (2014) CODEX-aligned dietary fiber definitions help to bridge the 'fiber gap'. Nutr J 13, 34 .

6. Bonnema AL, Kolberg LW, Thomas W, et al. (2010) Gastrointestinal tolerance of chicory inulin products. J Am Diet Assoc 110, $865-868$.

7. Mensink MA, Frijlink HW, van der Voort Maarschalk K, et al. (2015) Inulin, a flexible oligosaccharide II: review of its pharmaceutical applications. Carbohydr Polym 134, 418-428.

8. Mensink MA, Frijlink HW, van der Voort Maarschalk K, et al. (2015) Inulin, a flexible oligosaccharide I: review of its physicochemical characteristics. Carbohydr Polym 130, 405-419.

9. Niness KR (1999) Inulin and oligofructose: what are they? J Nutr 129, Suppl. 7, 1402S-1406S.

10. Maurer AD, Eller LK, Hallam MC, et al. (2010) Consumption of diets high in prebiotic fiber or protein during growth influences the response to a high fat and sucrose diet in adulthood in rats. Nutr Metab (Lond) 7, 77.

11. Cani PD, Dewever C \& Delzenne NM (2004) Inulin-type fructans modulate gastrointestinal peptides involved in appetite regulation (glucagon-like peptide-1 and ghrelin) in rats. BrJ Nutr $\mathbf{9 2}, 521-526$.

12. Cani PD, Neyrinck AM, Maton N, et al. (2005) Oligofructose promotes satiety in rats fed a high-fat diet: involvement of glucagon-like peptide-1. Obes Res 13, 1000-1007.

13. Beylot M (2005) Effects of inulin-type fructans on lipid metabolism in man and in animal models. BrJ Nutr 93, Suppl. 1, S163-S168.

14. Panchal SK, Poudyal H, Iyer A, et al. (2011) High-carbohydrate, high-fat diet-induced metabolic syndrome and cardiovascular remodeling in rats. I Cardiovasc Pharmacol 57, 611-624.

15. Coudray C, Tressol JC, Gueux E, et al. (2003) Effects of inulintype fructans of different chain length and type of branching on intestinal absorption and balance of calcium and magnesium in rats. Eur J Nutr 42, 91-98.

16. Roberfroid MB (2007) Inulin-type fructans: functional food ingredients. J Nutr 137, 2493S-2502S.

17. Poudyal H, Panchal S \& Brown L (2010) Comparison of purple carrot juice and beta-carotene in a high-carbohydrate, high-fat diet-fed rat model of the metabolic syndrome. Br J Nutr $\mathbf{1 0 4}$, 1322-1332.

18. Poudyal H, Panchal SK, Ward LC, et al. (2013) Effects of ALA, EPA and DHA in high-carbohydrate, high-fat diet-induced metabolic syndrome in rats. J Nutr Biochem 24, 1041-1052.

19. de Simone G, di Lorenzo L, Costantino G, et al. (1988) Echocardiographic indexes of left ventricular contractility. Effect of load manipulation in arterial hypertension. Jpn Heart J 29, 151-160.

20. Kumar SA, Magnusson M, Ward LC, et al. (2015) Seaweed supplements normalise metabolic, cardiovascular and liver responses in high-carbohydrate, high-fat fed rats. Mar Drugs 13, 788-805.

21. Delmée E, Cani PD, Gual G, et al. (2006) Relation between colonic proglucagon expression and metabolic response to oligofructose in high fat diet-fed mice. Life Sci 79, 1007-1013.

22. Adam CL, Williams PA, Garden KE, et al. (2015) Dosedependent effects of a soluble dietary fibre (pectin) on food intake, adiposity, gut hypertrophy and gut satiety hormone secretion in rats. PLOS ONE 10, e0115438.

23. Folch J, Lees M \& Sloane Stanley GH (1957) A simple method for the isolation and purification of total lipides from animal tissues. J Biol Chem 226, 497-509.

24. Poudyal H, Panchal SK, Waanders J, et al. (2012) Lipid redistribution by alpha-linolenic acid-rich chia seed inhibits stearoyl-CoA desaturase- 1 and induces cardiac and hepatic protection in diet-induced obese rats. $J$ Nutr Biochem $\mathbf{2 3}$, $153-162$

25. Sagher FA, Dodge JA, Johnston CF, et al. (1991) Rat small intestinal morphology and tissue regulatory peptides: effects of high dietary fat. Br J Nutr 65, 21-28.

26. Kerem M, Salman B, Pasaoglu H, et al. (2008) Effects of microalgae Chlorella species crude extracts on intestinal adaptation in experimental short bowel syndrome. World $J$ Gastroenterol 14, 4512-4517.

27. Roberfroid M (1993) Dietary fiber, inulin, and oligofructose: a review comparing their physiological effects. Crit Rev Food Sci Nutr 33, 103-148.

28. Jacobsen R, Lorenzen JK, Toubro S, et al. (2005) Effect of short-term high dietary calcium intake on 24-h energy expenditure, fat oxidation, and fecal fat excretion. Int $J$ Obes (Lond) 29, 292-301.

29. Friedrich M, Petzke KJ, Raederstorff D, et al. (2012) Acute effects of epigallocatechin gallate from green tea on oxidation and tissue incorporation of dietary lipids in mice fed a highfat diet. Int J Obes (Lond) 36, 735-743.

30. Shin AC, Zheng H, Townsend RL, et al. (2013) Longitudinal assessment of food intake, fecal energy loss, and energy expenditure after Roux-en-Y gastric bypass surgery in highfat-fed obese rats. Obes Surg 23, 531-540.

31. Westerterp KR, Smeets A, Lejeune MP, et al. (2008) Dietary fat oxidation as a function of body fat. Am J Clin Nutr 87, 132-135.

32. Delzenne NM, Cani PD \& Neyrinck AM (2007) Modulation of glucagon-like peptide 1 and energy metabolism by inulin and oligofructose: experimental data. J Nutr 137, 2547S-2551S.

33. Parnell JA \& Reimer RA (2012) Prebiotic fibres dosedependently increase satiety hormones and alter 
Bacteroidetes and Firmicutes in lean and obese JCR:LA-cp rats. Br J Nutr 107, 601-613.

34. Verhoef SP, Meyer D \& Westerterp KR (2011) Effects of oligofructose on appetite profile, glucagon-like peptide 1 and peptide YY3-36 concentrations and energy intake. Br J Nutr 106, 1757-1762.

35. Chaudhri OB, Salem V, Murphy KG, et al. (2008) Gastrointestinal satiety signals. Annu Rev Physiol 70, 239-255.

36. Slavin J (2013) Fiber and prebiotics: mechanisms and health benefits. Nutrients 5, 1417-1435.

37. Kaur N \& Gupta AK (2002) Applications of inulin and oligofructose in health and nutrition. J Biosci 27, 703-714.

38. Akalin AS \& Erisir D (2008) Effects of inulin and oligofructose on the rheological characteristics and probiotic culture survival in low-fat probiotic ice cream. J Food Sci 73, M184-M188.

39. Little TJ, Horowitz M \& Feinle-Bisset C (2007) Modulation by high-fat diets of gastrointestinal function and hormones associated with the regulation of energy intake: implications for the pathophysiology of obesity. Am J Clin Nutr 86, 531-541.

40. Park JH, Kwon OD, Ahn SH, et al. (2013) Fatty diets retarded the propulsive function of and attenuated motility in the gastrointestinal tract of rats. Nutr Res 33, 228-234.

41. Roberfroid MB \& Delzenne NM (1998) Dietary fructans. Annu Rev Nutr 18, 117-143.

42. Lobo AR, Filho JM, Alvares EP, et al. (2009) Effects of dietary lipid composition and inulin-type fructans on mineral bioavailability in growing rats. Nutrition 25, 216-225.

43. Madden JA \& Hunter JO (2002) A review of the role of the gut microflora in irritable bowel syndrome and the effects of probiotics. Br J Nutr 88, Suppl. 1, S67-S72.

44. Veereman G (2007) Pediatric applications of inulin and oligofructose. J Nutr 137, 2585S-2589S.

45. Zhang C, Yin A, Li H, et al. (2015) Dietary modulation of gut microbiota contributes to alleviation of both genetic and simple obesity in children. EBioMedicine 2, 966-982.

46. Brun P, Castagliuolo I, Di Leo V, et al. (2007) Increased intestinal permeability in obese mice: new evidence in the pathogenesis of nonalcoholic steatohepatitis. Am J Physiol Gastrointest Liver Physiol 292, G518-G525.

47. Cani PD, Bibiloni R, Knauf C, et al. (2008) Changes in gut microbiota control metabolic endotoxemia-induced inflammation in high-fat diet-induced obesity and diabetes in mice. Diabetes 57, 1470-1481.

48. DiBaise JK, Frank DN \& Mathur R (2012) Impact of the gut microbiota on the development of obesity: current concepts. Am J Gastroenterol Suppl 1, 22-27.

49. Ghanim H, Abuaysheh S, Sia CL, et al. (2009) Increase in plasma endotoxin concentrations and the expression of Toll-like receptors and suppressor of cytokine signaling-3 in mononuclear cells after a high-fat, high-carbohydrate meal: implications for insulin resistance. Diabetes Care 32, 2281-2287.

50. Stenman LK, Waget A, Garret C, et al. (2014) Potential probiotic Bifidobacterium animalis ssp. lactis 420 prevents weight gain and glucose intolerance in diet-induced obese mice. Benef Microbes 5, 437-445.

51. Griffiths EA, Duffy LC, Schanbacher FL, et al. (2004) In vivo effects of bifidobacteria and lactoferrin on gut endotoxin concentration and mucosal immunity in Balb/c mice. Dig Dis Sci 49, 579-589.

52. Rodes L, Saha S, Tomaro-Duchesneau C, et al. (2014) Microencapsulated Bifidobacterium longum subsp. infantis ATCC 15697 favorably modulates gut microbiota and reduces circulating endotoxins in F344 rats. Biomed Res Int 2014, 602832.

53. Amar J, Chabo C, Waget A, et al. (2011) Intestinal mucosal adherence and translocation of commensal bacteria at the early onset of type 2 diabetes: molecular mechanisms and probiotic treatment. EMBO Mol Med 3, 559-572.
54. Janssen P, Rotondo A, Mule F, et al. (2013) Review article: a comparison of glucagon-like peptides 1 and 2. Aliment Pharmacol Ther 37, 18-36.

55. Cani PD, Possemiers S, Van de Wiele T, et al. (2009) Changes in gut microbiota control inflammation in obese mice through a mechanism involving GLP-2-driven improvement of gut permeability. Gut 58, 1091-1103.

56. Nilsson A, Ostman E, Preston T, et al. (2008) Effects of GI vs content of cereal fibre of the evening meal on glucose tolerance at a subsequent standardized breakfast. Eur J Clin Nutr 62, 712-720

57. Luo J, Rizkalla SW, Alamowitch C, et al. (1996) Chronic consumption of short-chain fructooligosaccharides by healthy subjects decreased basal hepatic glucose production but had no effect on insulin-stimulated glucose metabolism. Am J Clin Nutr 63, 939-945.

58. Lee KU, Park JY, Kim CH, et al. (1996) Effect of decreasing plasma free fatty acids by acipimox on hepatic glucose metabolism in normal rats. Metabolism 45, 1408-1414.

59. Stanhope KL \& Havel PJ (2008) Fructose consumption: potential mechanisms for its effects to increase visceral adiposity and induce dyslipidemia and insulin resistance. Curr Opin Lipidol 19, 16-24.

60. Weisberg SP, McCann D, Desai M, et al. (2003) Obesity is associated with macrophage accumulation in adipose tissue. J Clin Invest 112, 1796-1808.

61. Damaso AR, do Prado WL, de Piano A, et al. (2008) Relationship between nonalcoholic fatty liver disease prevalence and visceral fat in obese adolescents. Dig Liver Dis 40, 132-139.

62. Fabbrini E, Sullivan S \& Klein S (2010) Obesity and nonalcoholic fatty liver disease: biochemical, metabolic, and clinical implications. Hepatology 51, 679-689.

63. Hietbrink F, Besselink MG, Renooij W, et al. (2009) Systemic inflammation increases intestinal permeability during experimental human endotoxemia. Shock 32, 374-378.

64. Lau WL, Kalantar-Zadeh K \& Vaziri ND (2015) The gut as a source of inflammation in chronic kidney disease. Nephron 130, 92-98.

65. Huang EY, Leone VA, Devkota S, et al. (2013) Composition of dietary fat source shapes gut microbiota architecture and alters host inflammatory mediators in mouse adipose tissue. JPEN J Parenter Enteral Nutr 37, 746-754.

66. Gregor MF \& Hotamisligil GS (2011) Inflammatory mechanisms in obesity. Annu Rev Immunol 29, 415-445.

67. Barbaro NR, Fontana V, Modolo R, et al. (2015) Increased arterial stiffness in resistant hypertension is associated with inflammatory biomarkers. Blood Press 24, 7-13.

68. Pietri P, Vyssoulis G, Vlachopoulos C, et al. (2006) Relationship between low-grade inflammation and arterial stiffness in patients with essential hypertension. J Hypertens 24, 2231-2238.

69. Juskewitch JE, Knudsen BE, Platt JL, et al. (2012) LPS-induced murine systemic inflammation is driven by parenchymal cell activation and exclusively predicted by early MCP-1 plasma levels. Am J Patbol 180, 32-40.

70. Kararigas G, Dworatzek E, Petrov G, et al. (2014) Sex-dependent regulation of fibrosis and inflammation in human left ventricular remodelling under pressure overload. Eur J Heart Fail 16, 1160-1167.

71. Nicoletti A \& Michel JB (1999) Cardiac fibrosis and inflammation: interaction with hemodynamic and hormonal factors. Cardiovasc Res 41, 532-543.

72. Dietitians Association of Australia (1983) For the public: Smart Eating for you: Nutrition Information A-Z: Fibre. http://daa.asn. au/?page_id $=800$.

73. Reagan-Shaw S, Nihal M \& Ahmad N (2008) Dose translation from animal to human studies revisited. FASEB J 22, 659-661. 